\title{
浅论电力安全监察管理的现状及创新理念
}

郑瑞峰 张慧杰

国网内蒙古东部电力有限公司

DOI:10.32629/hwr.v2i11.1708

[摘要] 随着社会生产力的进步和发展, 电力资源对社会经济发展的影响也越来越大, 而电力安全监察工作本身在电力供应 工作中占据着重要的地位。为了给监察系统运行的安全性和稳定性提供保障, 同时为电力供应的质量奠定基础, 就需要使用科 学合理的措施,为安监工作实施的效果提供保障。基于此, 本文首先对电力安全监察管理进行概述,其次分析了电力安全监察管 理的现状,最后论述了电力安全监察管理的创新理念, 以期和同行进行交流。

[关键词] 电力安全; 监察管理; 现状; 创新理念

随着社会的不断发展进步, 电力安全监察工作逐渐受到 社会的高度重视, 大部分企业在电力安全监察工作中有自身 独特的监察理念, 并将其严格落实到电力监察实际工作中, 但就实际情况来看, 当前电力安全监察工作仍有待进一步完 善, 因此加大力度探讨电力安全监察工作的创新发展策略, 对于电力企业的综合发展具有重要的现实意义。

\section{1 电力安全监察管理概述}

电力安全监察管理主要指的是, 电气企业中电力安全监 察部门的工作人员, 严格按照国家有关的法律和法规, 将有 关规定和标准贯彻到企业或者行业内部有关生产和管理部 门之中, 同时对其实施监督和检查工作的一种活动。该监察 体系主要是由电力企业负责安全监察的有关部门和人员构 成的, 同时根据电力发展的具体需求, 不断的对该体系结构 进行完善, 在合理使用电力企业自身的监察体系的基础上, 能够有效的监察电力生产以及有关建设活动, 同时也可以为 领导层在实施综合性安全管理工作的过程中提供辅助。

\section{2 电力安全监察工作现状}

2.1 电力安全监察的现状分析 (1) 电力企业安全监察管 理工作效率不高。现阶段, 我国的各大公司纷纷开始重视和 关注信息化建设工作, 为了达到信息化办公的目标, 各个公 司都在不断的研究和发展信息化建设工作。电力企业的整体 信息化处于较低的水平,公司的信息化共享水平还不能够符 合当前工作的需求, 计算机管理系统的应用仍然存在着较多 的不合理之处, 管理系统不能够在第一时间发现生产过程中 存在的缺陷, 并且没有建立计算机系统的安全隐患应急预案, 因此导致计算机系统无法检测到生产管理过程中的漏洞, 该 系统的运用不能够及时的对安全隐患进行解除, 从而大幅度 的减小了工作的效率和质量。(2) 电力企业的设备存在质量 问题。目前, 随着电力设备技术水平的不断提升, 电力企业要 在第一时间更新电力设备。但是在实际的情况中, 因为电力 设备的更新需要花费一定的成本费用, 电力企业无法更新所 有需要更新的设备, 所以, 部分旧设备和新设备之间依然具 有无法兼容的问题, 在一定程度上加大了工作的难度。此外, 旧设备的运用加大了工作中的安全隐患, 安全装置也具有老
化的质量问题, 导致电力企业在生产运营的过程中存在着一 定的安全隐患。(3) 监察人员的综合素质较低。安全监察管 理工作具有一定的难度, 要求工作人员具备一定的综合素质, 工作人员的综合素质对最终的工作效果产生着直接的影响。 我国电力企业的安全监察工作人员的整体素质仍然处于较 低的水平, 安全监察工作人员的专业知识仍然不扎实, 此外, 部分新人的专业知识虽然比较扎实, 但是其工作经验不充足, 对后期工作的实施产生着重要的影响。安全监察管理工作的 实施不断会涉及到安全保障体系, 同时也会涉及到监督管理 体系, 对安全监察部门具有一定的要求。所以, 电力企业要充 分的认识和了解这个情况, 进而有效的提升监察工作人员的 综合素质。

\section{2 电力安全监察的目标分析}

就当前电力安全监察工作的基本目标来看, 其主要是以 简单话语来体现安全监察理念, 这一理念与安全监察以及安 全预防等内容存在密切的联系, 为全面提高电力安全监察工 作效率和质量, 需要集成数年来电力安全监察的思想、工作 经验和工作方法, 并积极借鉴相关先进思想, 取其精华, 深化 电力安全监察工作的思想, 便于工作人员理解和记忆, 以推 进电力安全监察工作的顺利开展。

\section{3 电力安全监察管理的创新理念}

3.1 实施电力行业办公自动化

随着网络信息化的不断发展和完善, 构建一个完善的电 力行业现代化的计算机网络系统, 促进各个电力企业之间的 信息共享目标的实现, 促进电力企业内部信息的充分交流和 互通, 保障电力安全监察工作的高效性和科学性。利用已经 建设的电力信息系统, 有效的提高电力管理公务处理工作的 效率和质量, 促进办公效率的提升, 同时也可以加强安全风 险的管理和控制工作。

\section{2 健全安全监察工作体系}

为推进电力安全监察工作的创新发展, 应当结合当前电 力安全监察的实际情况, 电力安全监察工作队伍始终占据着 主体地位, 因此加强电力安全监察队伍建设是非常必要的。 在健全安全监察工作体系的过程中, 应当以监察队伍建设作 
为重点内容, 全面提高电力安全监察人员的综合素质, 以切 实提高电力安全监察工作效率, 以保证电力安全生产的顺利 实现。因此在电力安全监察工作的创新发展过程中, 应当积 极采取有效措施健全安全监察工作体系, 并将其合理应用于 实践活动中, 对带那里安全监察工作进行准确评估, 明确电 力运行过程中的潜在安全隐患, 并制定科学合理的电力安全 监察工作方案, 将电力安全监察制度严格落实到具体工作中, 推进电力安全监察工作的高效开展, 从而切实提高电力运行 的可靠性和安全性。

\section{3 提高监察工作人员素质技能}

电力企业一直非常重视安全问题, 而监察人员管理者安 全工作。工作人员的技能和素质对安全管理工作的效果产生 着直接的影响。电力企业要充分的重视和关注安全管理工作, 同时要对安全管理工作人员进行重视。对企业人才培养制度 进行完善, 增强人才培养工作实施的力度。在平常的工作中, 可以通过聘请专业工作人员的方法, 为安全管理工作的实施 提供指导。也需要采用不同的方法, 为企业间员工的安全文 化交流提供支持。各企业之间也要积极的进行沟通和交流, 对彼此的长处进行借鉴, 对自身工作中的不足进行弥补。并 且要构建满足本企业具体的安全监察管理工作体系。

3.4 对现代化管理方式进行合理运用

为保证电力安全监察工作的顺利高效开展, 相关监察工 作人员应当坚持具体问题具体分析的原则, 从基层工作入手, 对电力运行各环节进行全面且细致的安全监察, 优化配置监 察工作中的人力物力资源, 提高资源利用效率, 最大程度上 满足电力企业在技术性和专业性上的要求, 全面提高电力安 全监察工作的有效性。在这一过程中, 电力企业应当结合自 身发展实际, 对现代化管理方式进行合理运用, 优化调整电 力安全监察工作方式, 明确电力安全监察工作计划, 积极开 展电力安全监察与管理系统的推广工作, 从而为电力安全监 察系统的安全高效运行提供可靠的保障, 切实提高电力服务 的质量和效率。

3.5 明确电力安全监察工作的研究方法

为进一步促进电力安全监察工作的创新发展, 应当明确 电力安全监察工作体系的基础上, 掌握好电力安全监察工作
的研究方法, 从而推进电力安全监察创新发展的顺利实现。 通过分析可知, 电力安全监察的创新发展策略研究方法主要 包含以下几个方面: 一是研究法, 此种方式不仅仅是从文献 研究入手, 而是以电力安全监察历史作为切入点, 在获取电 力安全监察工作相关历史文献后, 开展深入研究和全面分析, 进行提炼总结, 并在专题会议上提出, 请相关专家进行评定, 为电力安全监察工作的创新发展提供灵感源泉, 从而切实提 高电力安全监察工作创新发展的科学性和合理性。二是调查 法。调查法首先要制定详细的计划, 来进行调查的对象的目 前发展状况, 通过调查来找到其研究的话题潜在的问题。

3.6 更新电力安全监察体系

要按照具体工作的状况, 及时的更新电力监察体系, 防 止出现由于体系不满足工作需求, 而对工作的实施产生不利 影响的情况。在科学有效的更新电力安全监察体系的基础上, 保证电力安全监察工作顺利的开展, 同时为电力安全监察工 作实施的效果提供保障。

\section{4 结束语}

总而言之, 电力安全监察管理工作实施的效果, 对整个 电力系统的运行质量产生着重要的影响, 所以要充分的重视 电力监察安全管理工作, 以此为电力系统的运行质量提供保 障。在实际的情况中, 电力安全监察管理工作还存在着一系 列的问题, 例如: 电力安全监察管理工作效率不高等等, 因此 电力企业要针对这些问题, 对电力安全监察管理工作进行创 新, 以此保障电力安全监察工作的顺利实施, 从而提升电力 系统的运行质量, 从而促进自身综合实力的提升。

\section{[参考文献]}

[1]芦毅,赵思文,钟长明.电力安全监察管理现状及创新 理念分析[J].黑龙江科学,2017,8(05):148-149.

[2]田勇,孙晋华.浅谈电力安全监察管理的现状及创新 理念[J].工程技术:文摘版,2016,(12):59.

[3]李建荣.试论电力企业安全监察工作的价值及实践 [J].企业技术开发,2016,35(18):58.

[4]曹琴琴.新形式下电力安全监察工作的重点研究 [J]. 中国新技术新产品,2016,(06):48+50. 